\title{
PROPUESTA DE UN INSTRUMENTO PARA LA CALIFICACIÓN, REGISTRO Y CERTIFICACIÓN DE LOS CONSULTORES AMBIENTALES SEGÚN LA LEY ORGÁNICA DEL AMBIENTE 7554: CASO DE ESTUDIO DE COSTA RICA
}

\author{
Roberto Quirós Vargas ${ }^{1}$
}

Recibido: 06/10/2017

\author{
RESUMEN
}

Aprobado: 06/12/2017

En esta investigación se comprueba que Costa Rica carece de un instrumento para la calificación y registro de los consultores ambientales que sea normalizado, sistematizado y que tenga la confianza y reconocimiento de la sociedad civil costarricense. Esta carencia origina, entre otras cosas, una calidad variable de las Evaluaciones de Impacto Ambiental. Como metodología para la recolección e indagación del objeto de estudio, se estudiaron las normas ISO (relativas a los procesos de calificación y registro) y se entrevistaron diferentes grupos de expertos a nivel local e internacional. El principal resultado de la investigación es el diseño de un esquema de calificación y registro de profesionales que desean ejercer la consultoría ambiental. Las competencias incluidas en el esquema para la evaluar a los profesionales que desean ejercer la consultoría ambiental fueron: educación, experiencia, formación y atributos personales. Estas competencias fueron evaluadas para las dos categorías de consultor ambiental consideradas: Coordinador de EIA y Especialista de EIA.

PALABRAS CLAVE: AMBIENTE, IMPACTO, CONSULTOR, CERTIFICACIÓN, REGISTRO, ESQUEMA, SETENA, ISO.

\section{ABSTRACT}

This research demonstrates that Costa Rica lacks of a standardized and systematized instrument for the qualification and registration of environmental consultants. The instrument is important to have the confidence and recognition of the Environmental Impacts Assessment by the Costa Rican civil society. This lack originates, among other issues, a variable quality of the Environmental Impact Assessments. As a methodology for the collection of data and other inputs of the study, Costa Rica legislation and ISO standards (i.e. ISO related to the accreditation and registration processes) were studied, and groups of experts (local and international) were interviewed to have inputs for the scheme. The main result of the research is the design of a qualification and registration scheme for the professionals who want to work as environmental consultants. The attributes included within the scheme to assess the work of environmental consultant were: education, experience, training and personal qualifications. These attributes were assessed for the two environmental consultants categories considered: EIA Coordinator and EIA Specialist.

KEYWORDS: ENVIRONMENT, IMPACT, CONSULTANT, CERTIFICATION, REGISTRATION, SCHEME, SETENA, ISO.

1 Universidad de Costa Rica, Escuela de Ingeniería Industrial; Código postal 11501-2060; San José, Costa Rica; roberto.quiros66@gmail.com 


\section{INTRODUCCIÓN}

Los problemas ambientales que enfrenta la humanidad empiezan a hacer su aparición desde épocas muy remotas. Esta problemática ambiental, foco de atención de las naciones del planeta, se ha visto favorecida por un crecimiento acelerado de la población mundial, cifra que supera las 100 millones de personas cada año. Durante los últimos 50 años, este crecimiento poblacional ha generado una serie de problemas, los cuales producen una mayor conciencia del impacto que el ser humano ha causado en el ambiente. Según Blanco (2004, p.6) "los problemas ambientales más serios que enfrenta la sociedad moderna son los de la contaminación y el agotamiento de los recursos naturales renovables y la forma en que estos están entrelazados".

Costa Rica, en concordancia con la tendencia mundial y en su afán por buscar soluciones a los problemas ambientales, su prevención y mitigación, ha desarrollado una amplia legislación ambiental, dentro de la que se destaca la Constitución de la República en el Artículo 50, en el cual se contempla que "Toda persona tiene derecho a un ambiente sano y ecológicamente equilibrado. Por ello, está legitimado para denunciar los actos que infrinjan ese derecho y para reparar el daño causado". Así mismo, en la Ley Orgánica del Ambiente No. 7574 del 25 de octubre de 1995, se establece en el Artículo 83, la creación de la Secretaría Técnica Ambiental (SETENA) como un órgano de desconcentración máxima del Ministerio del Ambiente Energía y Telecomunicaciones (MINAET). Esta institución tiene como propósito fundamental, entre otros, armonizar el impacto ambiental con los procesos productivos. Además, según el Artículo 17 de la Ley 7554, se establece que "Las actividades humanas que alteren o destruyan elementos del ambiente o generen residuos, materiales tóxicos o peligrosos, requerirán una evaluación de impacto ambiental" por parte de la SETENA. Dentro de las múltiples funciones de esta última, está la de analizar la actividad, obra o proyecto respecto a la condición ambiental del espacio geográfico que se propone. Para llevar a cabo esta labor, SETENA dispone de un instrumento científico y técnico titulado en la ley como: Evaluación de Impacto Ambiental (EIA), la cual le permite a la institución analizar los proyectos, obras o actividades, determinando los impactos, tanto negativos como positivos, que puedan ocasionar al ambiente, en conjunto con las actividades de control y seguimiento ambiental. Estos proyectos son catalogados de acuerdo a la severidad de los impactos que pueden causar al ambiente, y los profesionales encargados de calcular, medir y proponer medidas de mitigación a esos impactos son los consultores ambientales.

Conforme a estudios recientes y la apreciación de expertos, la SETENA no está cumpliendo a cabalidad el objetivo fundamental de su creación. Según Blanco, Campos, y Peralta (2007) el 67\% de las funciones otorgadas por ley a la SETENA se están incumpliendo. Por su lado, Rodríguez (2002) logra determinar atrasos significativos en la aprobación de los proyectos con duración de hasta 19 meses para lograr la viabilidad ambiental. Parte de las causas que influyen en la calidad de las EIA's, se atribuye a la gestión que realizan los consultores ambientales en su elaboración, por ejemplo: atrasos en la realización de los estudios, desconocimiento de la normativa ambiental, falta de conocimientos técnicos en la materia, entre otros.

En la revisión realizada a la normativa de la SETENA acerca de la función de los profesionales que realizan la consultoría ambiental, se observa que dentro de los requisitos, no especifican los conocimientos y experiencia en la temática ambiental que deben tener los consultores y responsables ambientales, para cumplir con las funciones que la ley les confiere. Así mismo, no existen mecanismos que por ley permitan calificar, registrar y certificar dichos requisitos. En resumen, el reglamento contempla que para ser consultor o responsable ambiental, debe cumplirse únicamente dos requisitos básicos: tener un título universitario sin distingo de profesión y estar incorporado al colegio profesional respectivo; y segundo, cancelar la cuota de inscripción vigente a la fecha de presentación del formulario de inscripción.

Como se puede observar, existe un gran vacío, ya que no se estipula qué competencias laborales, experiencia o conocimientos deberían tener estos profesionales, lo cual atenta contra la calidad de las EIA's. La evidencia de tales afirmaciones la encontramos en la misma Ley Orgánica 7554, la cual estipula en el Artículo 18, referente a la aprobación y costo de las EIA's, explícitamente que "la aprobación de las EIA's deberán gestionarse ante la SETENA, estas evaluaciones deberán ser realizadas por un equipo interdisciplinario de profesionales, inscritos y autorizados por la institución de conformidad con las guías elaboradas por ella".

Como se puede observar, aunque es un equipo interdisciplinario, la ley, en específico el artículo, no es explícito en las competencias que deberían tener los consultores ambientales para la elaboración de las EIA’s. Esta situación, es decir las decir las facilidades que ofrece la legislación para ser consultor ambiental, ha originado, entre otras, una afluencia masiva de profesionales, superando los 2.000 en el año 2015, quienes se inscriben como consultores ambientales, los cuales podrían no tener las competencias mínimas requeridas para ejercer tan importante función. Tampoco se indica en la normativa institucional el tipo de calificación que debe realizarse a los profesionales que ejercen la consultoría ambiental. 
Según estudio realizado por la Unión Mundial para la Conservación de la Naturaleza (UMNC) en el año 2006, se demuestra que la problemática planteada es tanto a nivel local como regional (países centroamericanos). En este estudio de la UMNC sobre las Evaluación de Impacto Ambiental en Centroamérica, determinó que los consultores ambientales no tienen los requisitos técnicos específicos para ejercer la consultoría ambiental (UMCN, 2006, p. 36). Este estudio establece la necesidad de contar con medios de control para el ejercicio y monitoreo de su labor.

Con base en lo expuesto, se concluye que la calidad de las EIA's no es la óptima, la cual se ve afectada por múltiples factores, entre los que se puede destacar para fines de esta investigación los asociados a los procesos de calificación y registro de los consultores ambientales, por cuanto no se hace una revisión exhaustiva y demostrativa de los requisitos mínimos que deben cumplir los profesionales que ejercen tan significativa labor, como son la formación académica, la preparación en temas específicos de la gestión ambiental, tales como: conocimiento de la legislación ambiental y conocimiento de los instrumentos para la evaluación de impactos ambientales, ni los años de experiencia en el ejercicio de tan significativa labor. Por lo tanto, se nota la importante y urgente necesidad de contar con medios al alcance de la institución (i.e. SETENA) y el país de disponer de un instrumento que le permita verificar $y$, por ende, garantizar los requisitos mínimos en cuanto a preparación y experiencia, entre otros, que deben tener los profesionales que ejercen la consultoría ambiental.

Cabe destacar, según se logró determinar en esta investigación, mediante una exhaustiva revisión bibliográfica, que a pesar de la transcendencia de la labor de los consultores ambientales tanto a nivel local como el internacional, no se cuenta, o por lo menos no hay evidencia, de esquemas de registro y certificación para profesionales que ejercen la consultoría ambiental que se puedan utilizar como modelo para el sistema que se diseña como producto de esta investigación.

Sin embargo, aunque no explícitamente para la labor de la consultoría ambiental, se logró encontrar elementos importantes en la Ley 8279 que establece la creación del Sistema Nacional de la Calidad, la cual contempla actividades para evaluar la conformidad de bienes y servicios para demostrar el cumplimiento de los requisitos reglamentarios, por ejemplo, los contemplados en la Ley Orgánica del Ambiente, entre otras, o de carácter voluntario como lo son las Normas ISO aplicables a estos bienes o servicios (Ley Nacional de la Calidad $8279,2002)$. Por lo tanto, esta Ley 8279, junto con los elementos mencionados, se utilizaron como referencia para el diseño del esquema de calificación y registro para el ejercicio de la consultoría ambiental en Costa Rica. En el diseño del esquema se específica que por ser una evaluación de la conformidad de cumplimiento de los atributos, es decir de las competencias de los profesionales que ejercen la consultoría ambiental, el ente que verifica y certifica esta condición debe ser un organismo de tercera parte (ente certificador), el cual, a su vez, debe estar acreditado ante el Ente Costarricense de Acreditación (ECA).

\section{METODOLOGÍA}

La metodología implementada para el análisis del objeto de estudio (i.e. calificación y registro de consultores ambientales) se divide en dos fases: a. la primera se refiere a la indagación de elementos teóricos y normativos en torno a los sistemas de calificación, registro, acreditación y certificación de personas, y entes que ejercen la consultoría ambiental; b. $y$ la segunda se refiere a la indagación y recopilación de información y variables claves que debe tener un esquema de registro y calificación de profesionales que ejercen la consultoría ambiental. Esta información se obtiene mediante la entrevista y encuesta a diferentes grupos de interés que tienen una participación (directa e indirecta) en la elaboración de las EIA’s y los esquemas de registro y calificación de profesionales que desempeñen o quieran desempeñar la labor de consultores ambientales.

Normas ISO para acreditación, certificacióny registro de organismosy personas: la Organización Internacional para la Estandarización (ISO, por sus siglas en inglés) contiene una gama muy amplia de normas, dentro de las que se distinguen las relacionadas con los esquemas de acreditación, certificación y registro de personas. Estas normas tienen como fin, entre otros, garantizar la estandarización y validez del instrumento mediante la transparencia en los procesos y la aceptabilidad en entes públicos y privados a nivel local e internacional. Estas normas y otras a las cuales se hará referencia en esta investigación tienen importancia significativa; ya que sobre ellas se fundamenta la propuesta del esquema. Es importante destacar que, aunque el esquema de calificación y registro tiene como fundamento teórico las normas ISO que son de carácter voluntario, el mismo tiene como marco regulatorio la legislación costarricense, como lo es la Ley 8279 que crea el Sistema Nacional de la Calidad, el cual, aunque no es explícito para la labor de la consultoría ambiental, sí es aplicable para la evaluación de la conformidad de los productos y servicios de la actividades vinculadas al desarrollo y demostración de la calidad.

En esta investigación se hace especial énfasis a la norma INTE - ISO/IEC 17024:2003 que contiene los requisitos generales para los organismos que certifican personas (consultores ambientales) que tienen como 
propósito evaluar conformidades para el ejercicio de una actividad. Según Bertrand (1997), la certificación "se define como la operación que asegura las competencias y habilidades de un individuo en relación con una norma formalizada". Por otro lado, Irigoin y Vargas (2002) definen las competencias laborales como el reconocimiento público, documentado, formal y temporal de la capacidad laboral demostrada por un trabajador, efectuado con base en la evaluación de sus competencias en relación con una norma sin estar necesariamente sujeto a la culminación de un proceso educativo.

Otra de las normas ISO utilizadas en la investigación es la Norma INTE-ISO/IEC 17011. Evaluación de la Conformidad. Esta norma tiene que ver con los procesos de acreditación de organizaciones que se encargan de acreditar entes que "evalúan conformidades". La certificación de consultores ambientales es una de las formas de asegurar que la persona certificada cumple los requisitos del esquema de registro y certificación. Esta norma internacional servirá de base para el reconocimiento de los organismos de certificación y sus esquemas, con el fin de facilitar su aceptación en los ámbitos nacional e internacional.

Por otro lado, la norma INTE - ISO/IEC 17024:2003 establece los requisitos que aseguran que los organismos de certificación que operan los esquemas de certificación de personas, trabajen en forma coherente, comparable y confiable; estableciendo la relación entre la norma ISO 17024 y la ISO 17011, en esta última, se define la acreditación como una "atestación de tercera parte relativa a un organismo de evaluación de la conformidad que manifiesta la demostración formal de su competencia para llevar a cabo tareas específicas de evaluación de la conformidad" (Figura 1). El organismo que lleva a cabo la acreditación se dice que es un "organismo con autoridad". Cabe destacar que la autoridad de un organismo de acreditación generalmente se deriva de los Gobiernos de los países. Tal es el caso de Costa Rica, según la Ley No. 8279 del 21 de mayo del 2002 que establece el Sistema Nacional de la Calidad, el cual estipula en su artículo No. 19 la creación del Ente Costarricense de la Calidad (ECA), como el ente autorizado para realizar la acreditación de organismos de evaluación de la conformidad.

Otras normas ISO utilizadas como referencia en esta investigación para el diseño del esquema para la calificación y el registro de los consultores ambientales, son: 1. Norma INTE - ISO / IEC 17000:2005 Evaluación de la conformidad - Vocabulario y principios generales. Esta norma internacional especifica los términos y las definiciones generales relacionadas con la evaluación de la conformidad, incluyendo la acreditación de los organismos de evaluación de la conformidad y el uso de la evaluación de la conformidad para facilitar el comercio; y 2. la Norma INTE - ISO 10019-2005. Directrices para la selección de consultores de sistemas de gestión de la calidad y la utilización de sus servicios. Esta norma pretende proporcionar orientación sobre los factores a tener en cuenta al seleccionar a un consultor de sistemas de gestión de calidad y la utilización de sus servicios. Es relevante destacar de esta norma, en su apartado 4.1.3, la forma para evaluar la competencia del consultor para lo cual se deberían considerar los siguientes aspectos: 1.atributos personales, 2.la educación pertinente, 3. los conocimientos $y$ las habilidades necesarias para cumplir con los objetivos globales de la organización respecto al sistema de gestión de calidad, y 4. la experiencia laboral y el comportamiento ético. Como se puede observar en la Figura 1, todas las normas ISO utilizadas para el diseño del esquema de registro y calificación de consultores ambientales como producto de esta investigación tienen una relación directa, lo cuales viene a soportar el marco estructural del esquema para garantizar su robustez. 
FIGURA 1

NORMAS ISO UTILIZADAS COMO REFERENCIA PARA EL DISEÑO DEL ESQUEMA

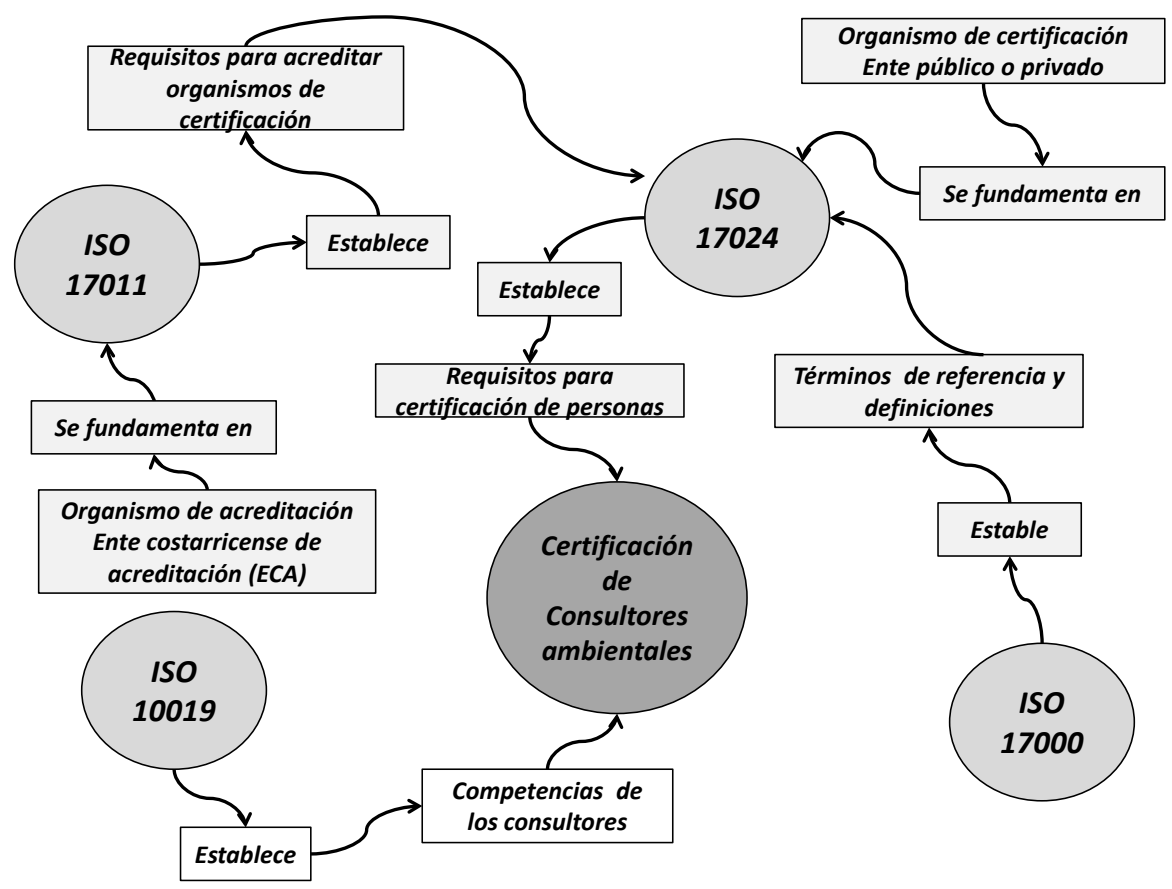

Fuente: Elaboración propia

Investigación y aportes de grupos de interés

Según la metodología empleada, la segunda fase del estudio consistió en la indagación del objeto del estudio (esquema de calificación y registro), para lo cual se tomó una muestra significativa de diferentes grupos involucrados (actores directos, participantes y afectados) que directa e indirectamente tienen relación con las EIA's y por ende con los esquemas de calificación y registro. La metodología se basa en diez etapas (Figura 2) que van desde la identificación de los grupos de interés, incluyendo el diseño y aplicación del instrumento, hasta un capítulo de conclusiones y hallazgos, donde se tomaron los insumos que junto con los elementos teóricos fueron la base para el diseño del esquema de calificación y registro. 
FIGURA 2

RESUMEN ESQUEMÁTICO DE LA METODOLOGÍA

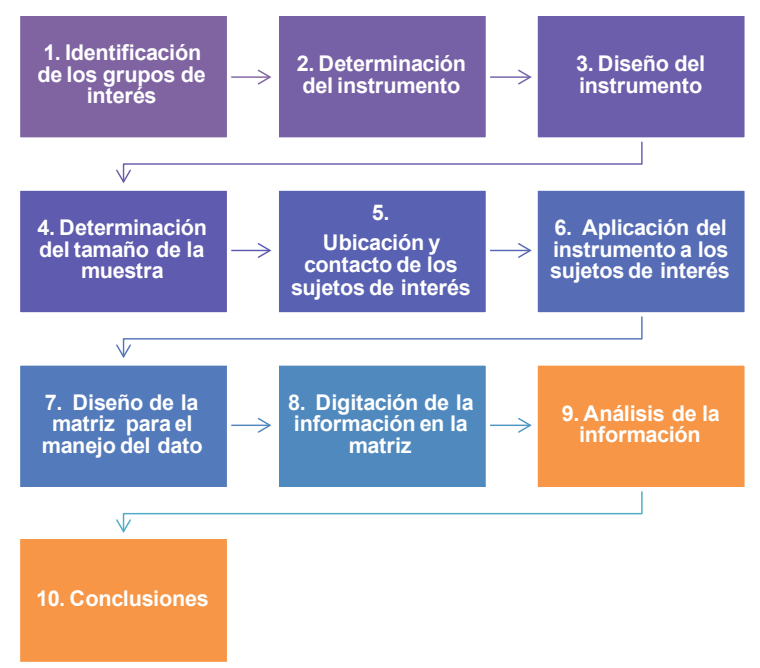

Fuente: Elaboración propia

Según Hernández, Fernández-Collado y Baptista. (2006), la recolección de los datos ocurre en los ambientes naturales y cotidianos de los participantes o unidades de análisis. Los grupos de interés (Figura 3) seleccionados para indagar el dato y recoger los insumos base del instrumento son: personal de la SETENA, expertos en temas de certificación y acreditación, consultores ambientales, fuentes internacionales (expertos de otros países), autoridades ambientales (ámbito costarricense) y representantes de los desarrolladores de proyectos (consultores en arquitectura e ingenieros). .

FIGURA 3

GRUPO DE INTERÉS PARA LA INDAGACIÓN DEL OBJETO DE ESTUDIO

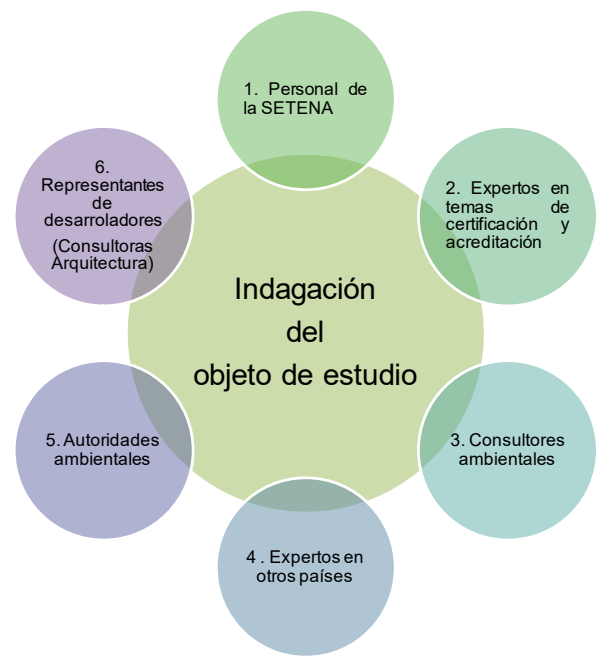

Fuente: Elaboración propia 
En total, se realizaron 57 encuestas, las cuales fueron distribuidas según el grupo de interés, para lo cual también se diseñaron diferentes formatos para la captura y análisis de datos según el grupo. En el caso del primer grupo, se entrevistaron ocho personas, este grupo incluía altos funcionarios, mandos medios y técnicos de la SETENA que participan en el proceso de EIA, excepto la Secretaria General que se incluyó en el grupo 5. En el grupo 2 , el de los expertos en temas de calificación, certificación, acreditación y temas asociados, se entrevistaron un total de diez y seis personas. Dentro de este grupo se incluyeron expertos de reconocidas instituciones costarricenses, tales como la Cámara de Industrias de Costa Rica, el Ente Costarricense de Acreditación (ECA), el Instituto Costarricense de Normalización (INTECO), el Instituto Nacional de Aprendizaje (INA), la Unión Internacional para la Conservación de la Naturaleza (UICN), la Fundación Centro de Gestión Tecnológica e Informática Industrial (CEGESTI), la Universidad de Costa Rica (UCR), el Colegio de Ingenieros y Arquitectos y otros expertos y expertas, quienes trabajan en puestos claves o como consultores o consultoras independientes a nivel nacional e internacional en temas ambientales propiamente, o asociados a los procesos de calificación, registro, certificación y acreditación. En el tercer grupo, se identificó y entrevistó una muestra de diez consultores ambientales y empresas consultoras inscritas en la SETENA, dentro de los criterios de selección para este grupo se consideraron aspectos tales como: recomendación de otros expertos, años de servicio, profesión, accesibilidad $y$ disponibilidad para ser entrevistados. En el cuarto grupo, se ubicaron expertos de otros países con los cuales se estableció contacto a través de la Internet. Similar al grupo 3, las personas contactadas en su momento laboraban para reconocidas instituciones ambientales homólogas de la SETENA tales como: el Department of Environment (DOE - Malasia), la Consellería de Medio Ambiente, Agua y Urbanismo de España, el Ente Nacional de Acreditación de España (ENA), la Asociación Española de la Calidad, la Red de Consultores Ambientales del Ecuador (RECAI), el Ministerio del Medio Ambiente de España, Certificación de Personas (CERPER de España), Departamentos relativos al medio ambiente de la Unión Europea, Universidades y centros de capacitación en Europa, National Registration Board for Personnel and Training (NRBPT of India) y el Quality Council of India (QCI). En el grupo cinco (autoridades ambientales), se incluyeron a personas que trabajaban en aquel momento para instituciones del Estado que tuvieran un nivel decisorio en materia ambiental y que, por ende, pudieran aportar elementos importantes en torno a la labor de los consultores ambientales. Dentro de este grupo se incluyó al Ministro de Competitividad, que en su momento tenía responsabilidad directa en la SETENA; una Exsecretaria y la Secretaria de la SETENA en aquel momento; el Presidente del Tribunal Ambiental Administrativo, órgano adscrito al MINAET y un fiscal ambiental de la Corte Suprema de Justicia de Costa Rica. El último grupo (grupo seis) estuvo conformado por los representantes de los desarrolladores de actividades, obras o proyectos ambientales. Este grupo generalmente son contratados por los desarrolladores (inversionistas) de actividades, obras o proyectos para desarrollen los proyectos (diseño, construcción y seguimiento), y son los responsables de dar seguimiento a la labor de los consultores ambientales. En total este grupo estuvo conformado por una muestra de diez empresas consultoras.

Como medio para la recolección de los datos se utilizó la entrevista. Según Hernández, et al, (2006), la entrevista se define como "una reunión para intercambiar información entre una persona (el entrevistador) y otra (el entrevistado)". En la entrevista, a través de preguntas y respuestas, se logra una comunicación y la construcción conjunta de significados respecto a un tema (Janesick, 1998). Por la naturaleza de la investigación, se utilizó lo que se conoce técnicamente como la entrevista estructurada, la cual se define, según Hernández et al. (2006) "como aquella en la cual el entrevistador realiza su labor con base en una guía de preguntas específicas y se sujeta exclusivamente a esta (el instrumento prescribe qué ítems se preguntarán y en qué orden)".

Para la investigación, se diseñaron un total de seis formatos de encuesta según el grupo de interés. Al igual que la conformación de los grupos, las preguntas para cada uno de los formatos fueron diseñadas según la experiencia de cada uno de los grupos y sujetos, el conocimiento acumulado en el tema a indagar y en función del aporte esperado de cada uno de ellos.

Referente al tamaño de la muestra, es importante señalar, para propósitos de esta investigación, la cantidad de sujetos que se entrevistaron, lo que viene a constituir el tamaño de la muestra. Esto se determinó con criterio de experto, tratando de buscar la representatividad de todos los grupos de interés que previamente se seleccionaron. Según Hernández et al (2006), "la muestra en el proceso cualitativo, es un grupo de personas, eventos, sucesos, comunidades etc., sobre el cual se habrán de recolectar los datos, sin que necesariamente sea representativo del universo o población que se estudia" (p. 562). Además señala Hernández et al. (2006) que en los estudios cualitativos el tamaño de la muestra no es importante desde una perspectiva probabilística, pues el interés del investigador no es generalizar los resultados de su estudio a una población más amplia; lo que se busca en la indagación cualitativa es profundidad. Por lo tanto, se pretende calidad en la muestra, más que en la cantidad. 
En cuanto a la metodología para el análisis de los datos, se utilizó lo recomendado por Hernández et al. (2006), el procedimiento más común para el análisis de los datos cualitativos es el que se denomina teoría fundamentada, lo cual significa que la teoría (hallazgos) va emergiendo fundamentada en los datos.

La metodología propiamente para el análisis de los datos de esta investigación se basó en las siguientes etapas: diseño en Excel de la matriz de análisis para cada una de los grupos de interés (en esta matriz, se presentan los elementos más relevantes indagados en las entrevistas); $y$ la identificación de tendencias, patrones y características relevantes por cada grupo de interés. Para esto, se analizaron las respuestas de cada uno de los sujetos entrevistados dentro de cada grupo y se ubicó dentro de la matriz de análisis. Esta metodología permitió identificar tendencias en torno de los temas de certificación, registro y calificación de consultores.

Una vez tabulados los datos, se identificaron oportunidades de mejora por cada grupo indagado, En el cuadro 1 resume los principales aportes de los seis grupos de interés entrevistados. 
1. Problemática asociada a la labor de los consultores ambientales y al sistema actual

2. Respecto al sistema actual de la SETENA para inscribir a los consultores.

3. Asociación de la labor de los consultores ambientales con la calidad de la EIA.

4. Criterios y requisitos mínimos (competencias) de los profesionales para ejercer la consultoría ambiental.

5. Categorización de las labores de los consultores ambientales.

6. Ente recomendado diseñar, implementar $\mathrm{y}$ administrar el sistema

7. Normas internacionales vinculadas a los procesos de registro y acreditación.

8. Beneficios esperados del instrumento para la calificación y registro de los consultores ambiéntales.

\section{Hallazgos}

Errores en la preparación de la EIA.

$>$ Omisiones en la documentación.

$>$ Falta de conocimientos en la preparación de la EIA.

Desconocimiento de la normativa ambiental

$>$ Atrasos en la obtención de las viabilidades (licencias) para la ejecución de las actividades, obras o proyectos.

$>$ Gran volumen de documentos rechazados, cuya causa es atribuida según la SETENA a EIA mal elaboradas.

$>$ Falta de preparación y conocimientos de los personeros de la institución encargados de revisar la EIA e instrumentos asociados.

$>\quad$ No es un registro es un listado.

> No hay criterios definidos para quienes ejercen la consultoría ambiental.

$>$ Es una labor muy técnica, no cualquier profesional la puede desempeñar.

$>\quad$ No se califican a los consultores por sus atributos, cualquier profesional puede ser consultor ambiental.

$>\quad$ No hay una sistemática para la calificación y registro.

$>\quad$ El proceso no está normalizado.

$>$ Es directa ya que son los consultores los que preparan la EIA.

$>$ Existen elementos subjetivos que de no ser manejados adecuadamente podrían afectar la calidad de la EIA.

$>$ Título universitario

Formación en temas ambientales

$>$ Experiencia

$>$ Atributos personales (ética)

$>$ Coordinadores de EIA

$>$ Especialistas en temas específicos

$>$ Asistentes de EIA

$>$ Integración de equipos de trabajos según el tipo de proyecto.

$>$ Dos posiciones: a. la SETENA o similar de carácter público y b. un ente privado (independiente).

> Para la certificación de los consultores: la norma INTE - ISO / IEC 17024.

$>$ Para la acreditación del ente que certifica: la norma INTE - ISO / IEC 17011

$>\quad$ Otras: ISO 17000, 9000 y 10019.

$>$ Reconocimiento internacional.

$>$ Credibilidad

$>$ Objetividad

$>$ Transparencia

$>$ Independencia

$>\quad$ Vinculación de lo voluntario (normas ISO) con lo normativo (Ley No. 8279) 
Como resultado de las anotaciones antes presentadas, se puede concluir que la calidad de la EIA es variable $y$ se ve afectada por múltiples factores, entre ellos se encuentra la labor que ejercen los consultores ambientales sobre la EIA e instrumentos asociados, la cual constituye el objeto de estudio esta investigación. Por lo tanto, es imprescindible para Costa Rica disponer de un instrumento de registro y certificación de los profesionales que ejerzan o deseen ejercer consultoría ambiental, el mismo se debe fundamentar en la legislación costarricense como marco jurídico (i.e. Ley Orgánica del Ambiente 7554) y las Normas ISO para garantizar la credibilidad, objetividad, transparencia e independencia, a su vez, que tenga el reconocimiento internacional.

\section{RESULTADOS}

El principal resultado como producto de esta investigación es el diseño del Esquema de registro y certificación de consultores ambientales para la SETENA. El Esquema fue diseñado con base en los elementos teóricos utilizados como referencia (i.e. normativa costarricense y las normas ISO) y el aporte de los diferentes grupos de interés que fueron considerados.

Etapas del Esquema: el esquema está conformado por tres procesos principales: el registro inicial, la renovación y la vigilancia (Figura 4). El registro inicial está conformado por las seis actividades que definen el proceso de registro desde que el profesional o persona interesada presenta la solicitud para ser evaluados hasta que es incorporado, certificado y registrado en la base de datos de carácter público. Específicamente, estas actividades son: presentación de la solicitud por parte del aspirante, evaluación, decisión sobre la evaluación, certificación $y$ registro. La vigilancia se refiere al conjunto de actividades para asegurar el cumplimiento de los profesionales certificados según las disposiciones generales del esquema de registro. La renovación se refiere a los requisitos para la renovación de la certificación de acuerdo con la norma de competencia y otros documentos pertinentes.

\section{FIGURA 4
ESQUEMA DE REGISTRO Y CALIFICACIÓN}

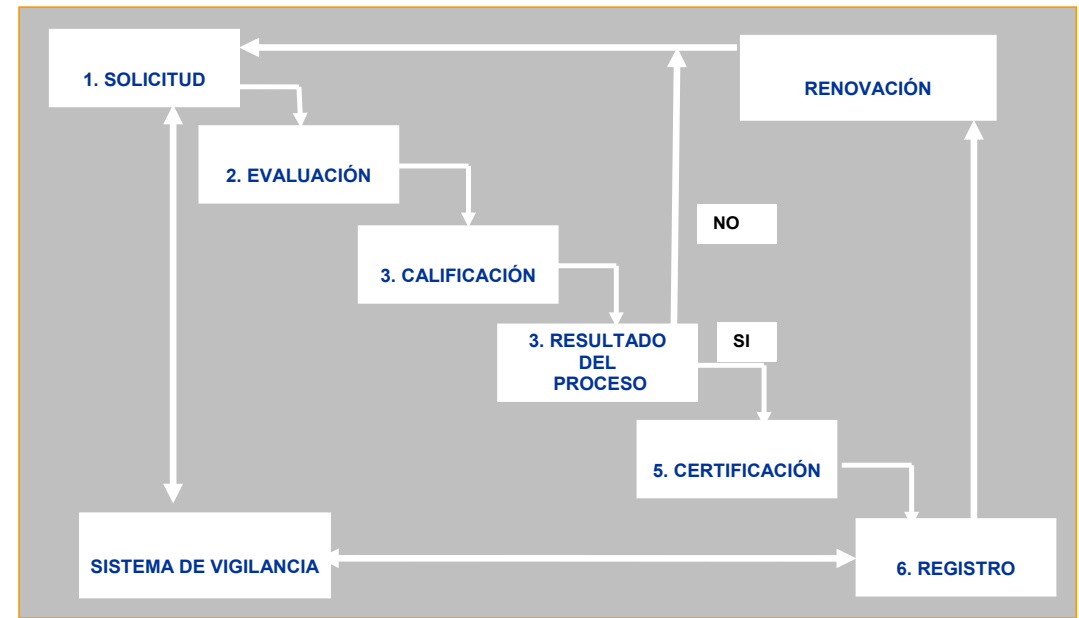

Fuente: Elaboración propia 
Actores principales: Los actores claves que participan en los procesos descritos son: el organismo de certificación (OC), los interesados (consultores ambientales), los desarrolladores de proyectos (públicos y privados), las instituciones de formación y capacitación, y los examinadores.

1. El organismo de certificación (OC): es el ente público o privado que lleva a cabo todo el proceso de calificación, certificación y registro. El OC es lo que se denomina un ente de tercera parte (ISO 17024), el cual tendrá la responsabilidad, entre otras, de certificar a los consultores ambientales e inscribirlos en un registro (i.e. base de datos) disponible al público.

2. Los interesados: este grupo está conformado por los profesionales que deseen desempeñar la consultoría ambiental, también son conocidos internacionalmente como prestadores de servicios ambientales. Estos aspirantes después de ser sometidos al proceso de calificación y registro por parte de la autoridad competente (OC), y confirmado el cumplimiento de la competencia son certificados $y$ registrados en la base de datos de acceso público.

3. Los desarrolladores: se distinguen dos sujetos: las personas físicas o jurídicas que vendrían a ser los inversionistas y las consultoras en arquitectura e ingeniería. Los primeros generalmente contratan a las consultoras en arquitectura e ingeniería para que desarrollen los proyectos, obras o actividades. Estos últimos, a su vez, contratan a profesionales especializados en el campo de la consultoría ambiental, para lo cual acuden a la base de datos de registro. Específicamente, estas empresas en arquitectura e ingeniería recurren a los consultores ambientales para que los asesoren en la ejecución de todo lo referente a los trámites de la SETENA conforme a la normativa ambiental del país, específicamente en lo referente a la elaboración de las EIA's e instrumentos asociados, o cualquier otro criterio según los requerimientos propios del proyecto y los términos de referencia (TR) dictados por la SETENA.

4. Instituciones de formación y capacitación: se refiere específicamente a los entes públicos y privados que brindan la capacitación a los profesionales que ejercen la consultoría ambiental. Estos entes prepararán los cursos en función de las necesidades previamente identificadas para fortalecer la competencia de los profesionales que ejercen la consultoría ambiental, y aumentar los conocimientos en torno a la temática ambiental y específicamente la EIA. Por ejemplo, dentro de los cursos de formación, se deben incluir normativa ambiental costarricense, determinación de riesgos ambientales y cursos de preparación en gestión ambiental, auditoría ambiental, entre otros. Estos cursos de formación podrán ser impartidos por universidades públicas y privadas, centros de enseñanza, institutos técnicos, etc. Estos entes tendrán un papel importante dentro del esquema, ya que la capacitación y formación es uno de los requisitos fundamentales dentro de la competencia de quienes ejercen la consultoría ambiental.

5. Los examinadores o evaluadores: son los profesionales que hacen la evaluación de la conformidad y son parte el OC. En caso de que sea necesario, se recomienda la posibilidad de realizar exámenes o cualquier otro tipo de prueba a criterio del OC y sus mecanismos para evaluar la competencia de los candidatos en áreas específicas de la EIA y sus temas asociados. Esto conlleva a la evaluación que según la norma es el proceso que evalúa el cumplimiento de una persona con los requisitos del esquema y que conduce a una decisión sobre la certificación (ISO 17024; ISO 17011). El examen vendría a ser el mecanismo probatorio que mide la competencia de un candidato por uno o varios medios tales como: escritos, orales, prácticos y de observación. Según la norma, "el examinador es la persona con calificaciones técnicas y personales pertinentes, que es competente para llevar a cabo o calificar el examen" (ISO 17024, p. 2).

\section{Otros Aspectos relevantes del Organismo Certificador (OC)}

El OC deberá efectuar la evaluación de la conformidad con el fin de demostrar el cumplimiento de los requisitos según la competencia. El concepto de competencia está asociado al conocimiento, habilidades y atributos personales. Por lo tanto, debe haber evidencia objetiva de que el candidato tiene los conocimientos necesarios adquiridos a través de su carrera base, experiencia, cursos de capacitación, formación y entrenamiento especializado. Para el caso del esquema de registro propuesto, se recomienda que el OC sea administrado por un ente de carácter público. En este caso, y según el aporte de expertos, el investigador recomienda que el OC dependa directamente del MINAET, por ser el ente rector de mayor jerarquía en materia ambiental a nivel de la República de Costa Rica. El MINAET designará un departamento u oficina para que lleve todo el proceso. Para garantizar la independencia y ganar credibilidad en el ámbito nacional e internacional, es importante que el ente que administre el esquema cumpla con todos los requisitos establecidos según la norma ISO 17024. Así mismo, 
esta condición tiene el reconocimiento del Estado costarricense, para poder ser acreditada por el ECA, el cual a su vez, sigue los lineamientos de la ISO 17011 para acreditar organismos que cumplen con la competencia y poder certificar personas. El OC debe estar estructurado de tal manera que ofrezca confianza a las partes interesadas e involucradas en el proceso de EIA, especialmente a los profesionales interesados en ejercer la consultoría ambiental, a los desarrolladores y sus representantes, a las instituciones del Estado, a la comunidad internacional y a la población en general, con respecto a su competencia, garantizando la imparcialidad, legalidad e integridad.

\section{El Comité de Certificación (CC)}

Para garantizar la competencia, imparcialidad, legalidad e integridad del Organismo Certificador, la norma ISO 17024 propone la creación del Comité de Certificación (CC), el cual será el responsable del desarrollo y mantenimiento del esquema de certificación y registro para cada tipo de certificación a ser considerada dentro del esquema. Este comité será conformado en forma equitativa e imparcial, por representantes de las partes significativamente involucradas. La conformación de este comité será decisión de la máxima autoridad política en materia ambiental, en este caso el MINAET quien a su vez, tendrá participación directa o a través de un representante. Un aspecto relevante es que este Comité deberá reunir a los diferentes sectores de la sociedad civil costarricense que tengan vínculos con la problemática ambiental, $y$ los procesos de certificación y acreditación, preferiblemente con conocimiento de la temática ambiental. Dentro de estos se pueden incluir: universidades, ministerios, organizaciones no gubernamentales, representantes de la autoridad ambiental, instituciones autónomas, cámaras privadas y representaciones internacionales.

Para lograr dicha representatividad, $y$ considerando tanto la normativa como la opinión de expertos en la materia, el investigador sugiere que este comité debe tener como mínimo cinco miembros dentro de los cuales se recomiendan:

$\checkmark$ Un representante del MINAET

$\checkmark$ Un representante de la SETENA

$\checkmark$ Un representante de los Colegios Profesionales

$\checkmark$ Un representante de las universidades públicas del país

$\checkmark$ Un representante de los sectores privados por ejemplo:

UCCAEP (Unión Costarricense de Cámaras y Asociaciones de la Empresa Privada).

Dentro de las funciones más relevantes del CC están: el veredicto final acerca de la incorporación de un nuevo miembro, la resolución de apelaciones y quejas por parte de los interesados, la revisión de futuras modificaciones a los requisitos de competencia establecidos, entre otras. También representa un órgano de carácter político con máxima autoridad y representación ante los poderes del estado y sus homólogos en temas de certificación, registro y acreditación de personas que están certificadas, actividades ejecutivas de planeamiento, seguimiento $y$ control del OC $y$ del esquema de registro.

Competencias a calificar en los consultores ambientales, las competencias incluidas en el esquema de registro y calificación de los consultores ambientales para garantizar la idoneidad de quienes ejercen o deseen ejercer la consultoría ambiental son: educación, formación, experiencia y atributos personales. Algunos aspectos generales de competencias están: 1. Educación, será necesario que el profesional aspirante posea el título de bachiller, licenciado, maestría o doctorado de una universidad acreditada en el sistema nacional de universidades costarricense, preferiblemente que estos títulos deberán tener afinidad con la temática ambiental y las específicas según los protocolos de la EIA, por ejemplo: ingeniería (civil, química, mecánica, industrial, etc.), arquitectura, biología, derecho, arqueología, sociología, entre otras. 2. Formación: cursos aprobados y capacitación en normativa ambiental costarricense e internacional, $y$ en temas de EIA y gestión ambiental según los contenidos de la EIA. Esta formación es un mecanismo para garantizar la actualización de los profesionales quienes teniendo una carrera base se mantengan actualizados en las diferentes temáticas asociadas a la EIA. 3. Experiencia: esta dependerá de la categoría para la cual estará optando el profesional. Para calificar la experiencia se tomó como referencia el Reglamento General sobre los Procedimientos de Evaluación de Impacto Ambiental y el Manual de Instrumentos Técnicos para el proceso de Evaluación de Impacto Ambiental, en el cual se identifican los trabajos que pueden ejercer los consultores para las categorías de Coordinador de EIA y Asistentes de EIA, y los Especialista de EIA. Según el tipo de trabajo (EIA's realizadas) se le asigna un valor para cada uno de ellos, por lo tanto, el aspirante será calificado en función del número de trabajos realizados durante un lapso de tres años u otro a determinar por el OC cuando esté en operación. 4. Atributos personales: para valorar esta competencia se sugiere concretarlas mediante pruebas de tipo psicométrico y de personalidad a los aspirantes para estas pruebas se recomienda recurrir al Servicio Civil quien es el órgano estatal en materia de contratación de empleados del sector público. 
Categoría de consultores: según el Procedimiento General de EIA de la SETENA se consideran las dos categorías de consultores ambientales: Coordinadores de EIA y Asistente de EIA, y los Especialista en temas específicos según los distintos protocolos requeridos para la elaboración de la EIA. Los Coordinadores de EIA y los Asistentes de EIA tienen como función principal el de asistir a los desarrolladores de actividades, obras o proyectos en los aspectos técnicos y en la preparación de las EIA`s y los trámites estipulados según el Procedimiento General de EIA y el Manual de EIA que rige en la institución (i.e. SETENA), entre otras funciones, además les corresponde llenar los formularios (D1 y D2) requeridos según el tipo de proyecto, presentar los formularios ante la SETENA, contratar a los Especialistas de EIA según los protocolos y dar seguimiento a las diferentes etapas del proyecto, y representar al desarrollador ante la SETENA en las diferentes etapas del proyecto, obra o actividad. Los Especialistas de EIA son los que realizan los estudios técnicos específicos, por ejemplo estudios de suelos, geología básica, biología, estudios arqueológicos, etc., según los diferentes protocolos $y$ los términos de referencia (TR) requeridos para cada proyecto conforme a lo requerimientos y el Procedimiento General para la Elaboración de las EIA`s de la SETENA; además, les corresponde preparar los informes del estudio técnico realizado y otra serie de funciones contenidos en el Manual de EIA.

Método recomendado para calificación de los atributos del profesional aspirante: el criterio recomendado para garantizar que un aspirante tiene la competencia para ejercer la consultoría ambiental se basa en un sistema de puntos. El puntaje total obtenido por un aspirante es el resultado de los puntos alcanzados como producto de la combinación de los cuatro factores claves señalados en el apartado anterior (educación, formación, experiencia $y$ atributos personales) excepto el de atributos personales que por su naturaleza de índole cualitativa no suma puntos a la valoración. El Organismo de Certificación (OC) deberá fijar un valor mínimo de calificación para garantizar la competencia mínima, el cual servirá de base para que el aspirante pueda ser certificado y registrado como consultor ambiental. La Ecuación 1 muestra en términos generales el procedimiento de calificación:

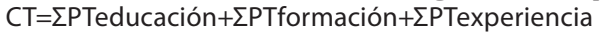

PTeducación=puntuación total por título obtenido

(Ecuación 1)

PTformación=puntuación total por formación

PTformación=puntuación total por experiencia

Educación: los grados académicos a ser reconocidos por el OC son: doctor, master, licenciado y bachiller. En la calificación de esta competencia el OC reconocerá solamente un título universitario, es decir el de mayor nivel que el aspirante haya alcanzado. Los títulos deberán ser debidamente reconocidos por el Comisión Nacional de Rectores (CONARE) que es la máxima autoridad a nivel costarricense en el reconocimiento de títulos y su homologación correspondiente en los casos en que se requiera; $y$ cualquier otra autoridad competente en materia de reconocimiento para la acreditación de títulos universitarios.

Experiencia: los criterios recomendados para la valoración dependerán de la categoría a la cual este optando el participante, es decir Coordinares y Asistentes de EIA o Especialistas de EIA. Para el caso de los Coordinadores y Asistentes de EIA el criterio recomendado se basa en la cantidad de evaluaciones que el aspirante haya realizado durante su vida profesional $y$ que los mismos sean verificables por medio de una evidencia confiable, en este caso la base de datos de la SETENA. Esta evaluación tiene como requisito que el aspirante haya realizado el proceso completo desde que presenta el documento de EIA, hasta que recibe la aprobación y por ende la viabilidad por parte de la SETENA para que pueda dar inicio la actividad, obra o proyecto. Los puntajes que se asignen estarán de conformidad a la complejidad del estudio realizado, por ejemplo, un Estudio de Impacto Ambiental aprobado con viabilidad ambiental tendría un valor de 100 puntos, un Plan de Gestión Ambiental tendría un valor de 70 puntos y una Declaración Jurada de Compromisos Ambientales tendría un valor de 30 puntos. También le OC puede otorgar al rubro de experiencia en otro tipo de trabajos que tienen que ver con la EIA y que están estipuladas en el reglamento como lo son las Auditorías Ambientales, en las cuales el consultor también ganaría puntos por experiencia.

Similarmente para los Especialistas de EIA se les asignará un valor de acuerdo a los estudios realizados según se contempla en el Reglamento General sobre las evaluaciones de EIA, por ejemplo, para el protocolo (i.e. estudio) de Ingeniería Básica se requiere un estudio de hidrología básica que lo debe realizar un Ingeniero Civil, este estudio tendría un valor de 100 puntos por cada estudio hasta un máximo de 5 estudios por período, etc.

Formación: se recomienda que la formación sea valorada en función del número de horas acumuladas por el aspirante en cursos de capacitación, seminarios u otros, debidamente reconocido por los organismos competentes, por ejemplo, el INA, el Servicio Civil, universidades públicas y otros. Por ejemplo, un curso de formación en Instrumentos de Evaluación de Impacto Ambiental que sea de más de 50 horas el candidato tendría un puntaje de 100; entre 24 y 49 horas 60 puntos, $y$ así sucesivamente. Los valores son recomendaciones para el OC, por lo tanto, los mismos serán debidamente revisados y adaptados por los responsables del OC que laboren en labores específicas de calificación de la formación. 


\section{CONCLUSIONES}

Costa Rica no dispone de un sistema que permita calificar y registrar sistemáticamente a los profesionales que ejercen la consultoría ambiental conforme a los criterios internacionalmente aceptados como lo son las normas ISO. Se recomienda que para lograr esta sistematización se siga el modelo del esquema de registro planteado en esta investigación que está en concordancia con lo establecido en la norma INTE - ISO / IEC 17024. El proceso de EIA y, por ende su calidad, se ve afectado por múltiples factores, uno de ellos, asociado al objeto de esta investigación, es la carencia de parámetros y criterios definidos para calificar y registrar a los profesionales que ejercen la consultoría ambiental. Se recomienda que las autoridades ambientales competentes diseñen los perfiles de los profesionales que ejercen la consultoría ambiental en función del área de interés y los campos de especialización requeridos para la realización de las evaluaciones de impacto ambiental y lo integren al instrumento propuesto.

El esquema de registro propuesto garantiza que los profesionales que ejercen la consultoría ambiental tengan las competencias mínimas y que, después de ser certificados, sean registrados en una base de datos de carácter público a disposición de todos los sectores involucrados en la Evaluación de Impacto Ambiental. Los diferentes actores participantes del proceso, especialmente la institución y los desarrolladores, son los que más severamente se ven afectados por las deficiencias en la preparación y presentación de los documentos por parte de los consultores ambientales, ya que esta situación, entre otras, origina atrasos para otorgar la viabilidad (licencia) ambiental.

Se recomienda, paralelamente al esquema de registro propuesto, que la autoridad ambiental revise los parámetros en la preparación de las evaluaciones de impacto ambiental de manera que se tenga una mayor estandarización en los documentos que se deben presentar para la realización de las evaluaciones de impacto ambiental. Conforme al Principio Precautorio, cuyo objetivo principal es prevenir, mitigar y controlar los efectos negativos que los seres humanos puedan causar en el medio ambiente, el instrumento de calificación y registro de los consultores ambientales propuesto se alinea con este importante principio, por cuanto, al garantizar las competencias mínimas de los que hacen la EIA, entonces los mismos tendrán criterios más sólidos y científicos para realizar su labor. A criterio del investigador y fundamentado en las normas internacionales ISO, específicamente la INTE - ISO / IEC 17024:2003, que establece en su apartado 4.2 e inciso a. que "el organismo de certificación debe estar estructurado de modo que ofrezca confianza en su competencia, imparcialidad e integridad en las partes interesadas". El esquema de calificación y registro debe ser administrado por un ente o institución que sea independiente de la institución responsable de llevar el proceso de evaluación, control y seguimientos del proceso de EIA, en este caso la SETENA. Siguiendo los lineamientos de las normas INTE - ISO 10019:2005 Directrices para la selección de consultores de sistemas de gestión de la calidad y utilización de sus servicios, así como de las entrevistas realizadas a los grupos de interés, se concluye que las competencias mínimas que deben tener los profesionales que ejercen la consultoría ambiental son: atributos personales (considera aspectos como la ética, responsabilidad y otras orientadas a características propias de la persona que ejerce una labor); la educación pertinente; los conocimientos y habilidades en la temática ambiental; $y$ la experiencia laboral.

Finalmente, es importante que el esquema para la calificación y registro de los consultores ambientales sea presentado y evaluado en el marco de la legislación costarricense, para que en caso de ser aprobado por las autoridades competentes, se hagan las reformas correspondientes a dicha legislación. Este último aspecto garantizará la transparencia, eficacia y aplicabilidad del instrumento al considerar los aspectos normativos de carácter voluntario, como son las normas ISO, con los aspectos normativos de la legislación costarricense.

carácter voluntario, como son las normas ISO, con los aspectos normativos de la legislación costarricense. 


\section{REFERENCIAS}

Bertrand, O. (1997). Evaluación y certificación de competencias y cualificaciones profesionales. París, Francia. Instituto Internacional de Planificación de la Educación (IIPE). UNESCO.

Blanco, M. (2004). Gestión ambiental: camino al desarrollo sostenible. San José, Costa Rica: Editorial UNED.

Blanco, F; Campos, J; Peralta, A. (2007). Formulación de un Modelo Organizacional para la Secretaría Técnica Nacional Ambiental. Tesis para optar por el grado de Licenciatura. San José, Costa Rica. Universidad de Costa Rica.

Constitución Política de la República de Costa Rica (1949). Derechos Individuales, enmiendas a los Artículos 46 y 50.

Hernández, S.; Fernández-Collado, C.; Baptista, P. (2006). Metodología de la Investigación. (4º ed.). México D.F.: Editorial Mc Graw Hill.

Irigoin, M.; Vargas, F. (2002). Competencia Laboral. Manual de conceptos, métodos y aplicaciones en el sector salud. Montevideo: Cinterfor / OIT.

ISOIEC 10019:2005 Directrices para la selección de consultores de sistemas de gestión de la calidad y la utilización de sus servicios.

ISO/IEC 17000:2004. Evaluación de la conformidad. Vocabulario y principios generales.

ISO/IEC 17011:2004. Evaluación de la conformidad. Requisitos generales para los organismos de acreditación que realizan la acreditación de organismos de evaluación de la conformidad.

ISO/IEC 17024:2012. Evaluación de la conformidad. Requisitos generales para los organismos que realizan certificación de personas.

Ley Orgánica del Ambiente 7554, (1995). Compendio de leyes que rigen la materia ambiental en la República de Costa Rica.

Manual de Instrumentos Técnicos para el Proceso de Evaluación de Impacto Ambiental (Manual de EIA), (2006).

Reglamento General sobre los Procedimientos de Evaluación de Impacto Ambiental (EIA), 2004. No 31849 -MINAE-SMOPT-MAG-MEIC.

Rodríguez, C. (2002). Evaluación de la Gestión Técnica de la Setena según la Ley Orgánica del Ambiente (7554) y los Reglamentos de la Setena. Tesis para optar por el grado de Licenciatura. San José, Costa Rica. Universidad de Costa Rica.

Unión Mundial para la Naturaleza (2006). Estudio Comparativo de los Sistemas de Evaluación de Impacto Ambiental en Centroamérica. San Salvador. El Salvador. 